\title{
Laboratory for Analysis of Microgrid with Real Time Simulation
}

\author{
A. B. Piardi ${ }^{1}$, R. B. Otto ${ }^{1}$, D. G. Sonoda ${ }^{1}$, F. C. Santos ${ }^{1}$, L.R.A Ferreira ${ }^{1}$ and R. A. Ramos ${ }^{2}$ \\ ${ }^{1}$ Itaipu Technological Park Foundation \\ LASSE - FPTI \\ Av. Tancredo Neves, 6731 Foz do Iguaçu -PR (Brazil) \\ Phone/Fax number: +0055 3576-7089, e-mail: lasse@pti.org,br \\ ${ }^{2}$ University of São Paulo \\ rramos@usp.br \\ (16) 3373-9348
}

\begin{abstract}
.
It becomes essential for Brazil the formation of critical mass and technological base in the microgrid subject and, mainly, the creation of an infrastructure that allows the accomplishment of research in this area of knowledge. Faced with this necessity, it was verified the viability of implementing a laboratory to perform analysis of microgrids systems in the actual premises of the Laboratory of Automation and Simulation of Electrical Systems, which is allocated in Itaipu Technological Park Foundation - Brazil dependencies. In the presented context, this work brings the description of the provisioned infrastructure whose the main feature will be to add flexibility of analysis through the joint application of real, emulated elements and realtime simulation platform, being at the same level of international reference laboratories as well as the fundamental aspects that provides the basis for your design and the possibilities of research envisaged from its implementation. The joint action of several researchers and enterprises will be stimulated and characterized as fundamental role for the transformation of this laboratory into a national reference in the study of microgrids serving as support for the electrical sector for the complete understanding of the new development trends of the future electrical power.
\end{abstract}

\section{Key words}

Microgrid; Real-Time Simulation Platform; Control Hardware-in-the-Loop; Power Hardware-in-the-Loop.

\section{Introduction}

The deregulation process of electrical power systems - that opened the way towards competitive markets with independent power producers [1] - added to the necessities demanded by industrialized economies characterized by the diversification of energy matrix as well as the increasing of the efficiency in the processes of generation and transport of electric power [2], provided the subsidies for the advent of Distributed Generation (DG) concept. For this work purposes, the DG can be characterized in general guidelines as the electrical energy production in distribution levels - that is, close to the consumer centers - through generators with relatively low nominal capacities [3], [4] which are predominantly based in renewable energy resources.

In order to obtain the potential benefits from DG fully harnessed, it was verified the necessity of a reformulation of the DPSs operation through the complete automation of its elements, giving rise to the concept of microgrids. Because it is a relatively new concept in the electrical power systems history, a detailed definition of microgrids is still under discussion in the technical forums. However, a microgrid can be generally described as a cluster of loads and Distributed Energy Resources (DERs) - which is composed by DGs and Energy Storage Systems (EESs) - in any arbitrary configuration, operated in coordination to reliably supply electricity to your customers with efficiency from the technical and economic viewpoints, which is connected to the host power system at the distribution level at a single point of connection [5] [6] [7]

To perform your tasks as expected, the microgrids are composed of complex electrical, control and communication systems that operate in a coordinated way [8]. For the fact that these systems can be considered a basic feature of future active DPSs [9] , and due to its inherent complexity of operation, it becomes mandatory to characterize their behavior and performance, both in the design phase as well as during their operation. In this sense, there is observed in a worldwide level a lot of testbed initiatives [7] [9] and laboratorial infrastructures [10] [11] dedicated to analyzing electrical systems with these characteristics, given that there are still a lot of challenges that involve this concept to be solved [6] [12] [13] .

Following the worldwide trend, it was verified the viability of implementing a laboratory to perform analysis of microgrids systems in the actual infrastructure of Laboratory of Automation and Simulation of Electrical Systems (LASSE - acronym in portuguese) in Itaipu Technological Park Foundation - Brazil (FPTI-BR, 
acronym in portuguese). Based in the simulation concepts of Control-Hardware-in-the-Loop (CHIL) and PowerHardware-in-the-Loop (PHIL) in joint application with real and emulated elements - which add flexibility of analysis and representation - the aim of the proposed infrastructure is to promote research in this area of knowledge in the national territory, in the same level observed in international centers, meeting the needs of Brazilian Electrical Sector (BES) from the technical and regulatory points of view.

In the context presented in the abovementioned paragraphs, the main objective of this work is to present aspects of the infrastructure to be implemented, as well as the main possibilities of research development from this one.

\section{Aspects and Approaches to Perform Microgrids Analyses}

The benefits and justifications of microgrid implementation are already consolidated in the literature [6] [9] . However, from the practical point of view, there are several challenges that need to be overlapped to make all the benefits coming from microgrids concept be fully harnessed. The general architecture of a microgrid, can be divided into three principal layers [7] [8] :

1) First layer: constituted by the power equipment (electrical system);

2) Second layer: constituted by the communication infrastructure, where the devices are interconnected by the cyber-physical network through communication protocols;

3) Third layer: constituted by the high-level control system, which contains the general controllers and Human-Machine Interfaces (HMIs).

In the first layer, the integration of several types of DERs and your controllers introduce a number of operational challenges that need to be studied in order to ensure the desired benefits of microgrid concept [6] [8] . For example, intermittent sources - like solar PV and wind needs a connection with energy storage systems to provide specified ancillary services. Moreover, the highpenetration of DERs introduce new issues in the current DPSs like: levels of reliability; economical and efficient operation; undesired (or even unstable) dynamic behaviors; voltage and frequency control; unintentional islanding; power quality concerns (due to the massive presence of electronically interfaced devices); discoordination as well as undesirable operation of protection functions; and bi-directional power flow in distribution feeders.

With respect to the challenges in the second layer, relevant challenges include the integration of different proprietary communication protocols, which can affect the time requirements of several applications of control loops. Another issue is related to the availability, reliability, and scalability that communication systems can offer to the microgrid environments [8], meeting the minimum requirements from the cybersecurity point of view.

In the third layer, there are some non-technical challenges for the implementation of microgrids controllers, such as regulatory rules of operation and business models for microgrids services. On the other hand, technical issues include coordination among different DERs and microgrids controllers to make a unified strategy of operation and control of the entire system [7] [9] , as well as the general improvement of dynamical behaviours to enhance the stability margins of the microgrid systems.

In order to identify and propose solutions to overcome the challenges previously pointed out, several methodologies and arrangements are proposed to evaluate the performance of devices and strategies of operation, control, and protection applied in microgrids systems - each one having its advantages and disadvantages [14] . In general guidelines, these arrangements can be separated into three different classes, how presented in Figure 1:

1) purely physical methodology;

2) purely simulated methodology;

3) an intermediate arrangement between the previous two methodologies.

The purely physical approach (Figure 1.d) uses only real devices to perform the desired analysis. By this reason, this approach presents the real behaviors of the devices that will operate in the microgrid, representing real operation concerns like signal noises and communication issues between the equipment. On the other hand, this approach has as the main limitations the impossibilities of analyses involving any operation scenarios especially the worst-cases - and the representation of different topologies and capabilities of the existent devices.

Unlike the purely physical methodology, the pure simulation methodology (Figure 1.a) - which is the simplest, cheaper and easiest-to-use approach - allows the evaluating of any scenario of microgrid operation, involving different topologies, devices, and events. However, the main limitation of this approach is the distance between the behaviors of the simulated microgrid and that verified in a real system, which is related to the problems of representation and modelling [14].

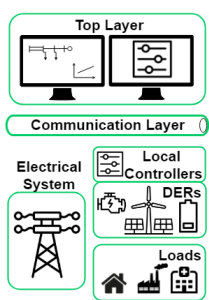

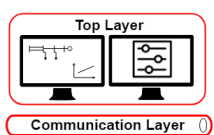

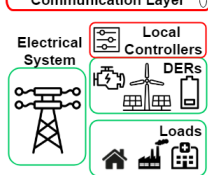

b)

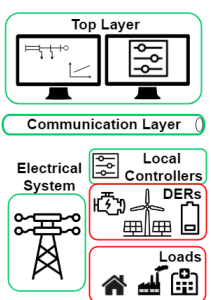

c)

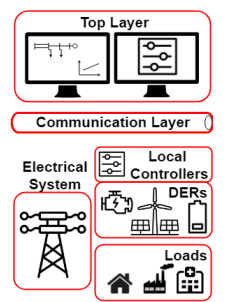

SIMULATED

INTERMEDIATE

PHYSICAL

Fig. 1 - Laboratorial methodologies 
The intermediate methodology to carry out microgrid analysis (Figure 1.b and Figure 1.c) joins the advantages of each methodology previously discussed. In this approach, a part of the evaluated system can be represented in a mathematical digital model that interfaces with real equipment of the electrical system. Thereby, this approach allows the analysis of real devices without the necessity to have a complete physical system. The main issue in this approach is on the choice of an appropriate interface between the simulation platform and real physical devices. In this case, it is recommended the use of a hardware device with capabilities of a hard-real-time system, in terms of deterministic and small time-step of the simulation, as is the case of Real-Time Simulators (RTSs) [18]. The most relevant RTSs for this application include the products of RTDS $®$ (Real-Time Digital Simulator), Opal-RT®, Typhoon HIL ${ }^{\circledR}$ and Speedgoat ${ }^{\circledR}$ manufacturers.

The great advantage of the RTS platforms is to allow the possibility to interface external devices - usually referenced as Device Under Test (DUT) - to the electrical power system being simulated, allowing the characterization of its behaviors under operational conditions very close to those encountered in the field, even under extreme and unusual conditions, in a controlled environment. This kind of simulation is usually referenced in technical literature as Hardware-in-the-Loop (HIL) and can be classified in two principal classes [15] [16] [17] :

1) Control-HIL (CHIL): in this case, that is represented by Figure 1.b, physical control elements or protection relays are interfaced with the RTS by the exchange of digital and/or analog sensing signals, commonly at low voltage levels. In this approach, it is also possible to use interfaces via communication protocols to perform the connection of DUTs and the RTS platform;

2) Power-HIL (PHIL): in this case, that is represented by Figure 1.c, power equipment such as inverters and electronic loads - are interfaced with the digital model of the electrical power system. In this application, a 4-quadrant (4Q) amplifier is required to obtain the signals from RTS and to provide/sink real and reactive powers in/from the DUT. In this approach, the device under test is a part of the simulated power system network. So, in the actual state of the art, it is reasonable to assert that PHIL is the most advanced and challenging approach in terms of simulation methodologies.

\section{Proposed Laboratorial Infrastructure}

In order to contribute with the subjects pointed out in the previous sections, this paper proposes a hybrid and flexible laboratorial platform to perform microgrid analyses. The hybrid and flexible terms are used due to the fact of the four approaches presented in Figure 1 be covered, allowing the possibility to perform analyses in simulated, intermediate and physical environments, separately or even in an integrated way.
The proposed platform will be integrated with the current premises of LASSE. This laboratory has been active in the BES for almost one decade, working mainly in research and development projects and providing services with high technical content for Itaipu Dam and utilities as well as enterprises of Brazil. The current infrastructure of the LASSE includes a full RTS platform - from RTDS ${ }^{\circledR}$ - to carry out CHIL studies in the subjects of protection and control of electric power systems. In this year, the infrastructure of the LASSE will be augmented to perform analyses using the PHIL methodology.

The laboratorial infrastructure that will be implanted in LASSE it will be characterized by various components, including power electronics interfaces (as for example, inverters and converters), generators with the capability to represent different primary sources (real and systems. The proposed infrastructure can be separated into four main groups, as described in the next subsection emulated), amplifiers, as well as monitoring and control

\section{A. Real Devices Grid}

As the proper name suggests, this group consist of a set of real sources and loads, like PV and synchronous generator, Battery Energy Storage System (BESS) and controllable and non-controllable loads of the laboratory, forming a real microgrid environment. Their goal is to perform analyses based on the pure physical methodology represented in Figure 1.d, involving commercial and/or tailor-made solutions. Like a real microgrid, this group can be operated in, both connected and islanded modes to the main grid. The user will be allowed to interact with the microgrid devices through a SCADA system, specially developed for this application.

\section{B. Emulated Devices Grid}

This group will be responsible to complement the physical devices, because it does not depend on external variables to modify your operation point (like insolation level in the PV solar panels, for example), enabling the repeatability and flexibility of the performed analyses. This subsystem will also allow the representation of different sources - like wind and fuel-cells generators, and gas microturbines - several ESSs technologies, Electrical Vehicles Chargers, and controllable loads, both with the possibility to perform triphasic or monophasic connections. The proposed structure also will allow the representation of a DC bus, which will be possible to connect each one of the emulated devices. The connection between $\mathrm{DC}$ and $\mathrm{AC}$ bus will be realized through a DC/AC converter. The users can control the emulated devices in two different ways: connection with RTS platform, or connection via the supervisory systems. As the "Real Devices Grid", this subsystem allows the performing of analyses using commercial or tailor-made solutions.

\section{PHIL Interface}

The power interface between RTS platform and physical devices will be fulfilled by "PHIL Interface" subsystem, 
where it will be allowed to carry out analyzes represented in Figure 1.c. This group basically consists of a set of 4Q amplifiers that makes the power balance between physical devices and simulated electric power system, allowing the representation of the behavior of devices that operate both in sink (consuming power) and source (providing power) modes, being possible - depending upon the application the representation of both AC and DC networks.

Regarding 4Q amplifiers, which can be classified as linear and nonlinear, they are critical to the stability and accuracy of simulation [15] . Linear 4Q amplifiers have as the main advantages of the high bandwidth and the fast response time (high slew rate), which enables the representation of fast transients (small time-step) in the simulated environment. However, their main disadvantage the reduced power capacity when it works in sink mode (some manufacturers inform the value of about $30 \%$ of the nominal capacity of the equipment when it operates in source mode). The cause of this behavior is the thermal limitations of yours internal electronic circuits, which are responsible to dissipate the consumed power when operating in source mode. On the other hand, non-linear 4Q amplifiers operate with rated power when working in sink mode. However, due to the presence of additional control circuitry, this kind of amplifiers shows reduced bandwidth and slow response times. This characteristic reduces the size of the time-step applied in the simulated environment, which is detrimental to the ability of representation of elements with fast transient behaviors.

In the last paragraph, it became clear that one class of $4 \mathrm{Q}$ amplifiers complements the other. So, in the provisioned infrastructure will be considered the two classes of amplifiers. These amplifiers will be used in two ways in the test environment, as shown in Figure 2. In the first one the amplifiers will operate like grid simulations, in which lof the simulated electrical power system under different scenarios, for the connection of the elements belonging to the "Real Devices Grid" and "Emulated Devices Grid" subsystems (Figure 2.a). In such application it is required the ability to operate in sink mode, without the need for representation of fast dynamics, being adequate the application of non-linear amplifiers. On the other hand, in the second application, the 4Q amplifiers will perform the interface between the "Real Devices Grid" and "Emulated Devices Grid" subsystems and mathematical models embedded in the RTS platform (Figure 2.b). These models can represent determined devices such as sources or loads with specific/particular characteristics, that is not addressed by the other devices present in the proposed infrastructure. In such application - depending upon the modeled device in the RTS platform - it is required high slew rates for the complete and adequate representation of modeled device's behavior, being adequate the use of linear amplifiers.

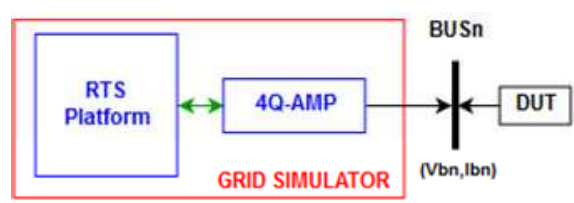

a)

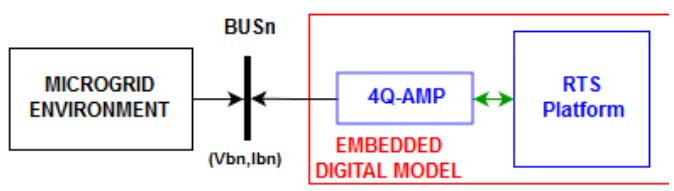

b)

Fig. 2 - Applications of $4 \mathrm{Q}$ amplifiers in proposed laboratorial infrastructure

\section{CHIL Interface}

Another approach to integrating the simulated and physical environments is the CHIL. This class of simulation is responsible to perform analyses in control and protection systems by the low signal level interface, as presented in Figure 1.b. This interface can be made through direct connection between RTS platform and the DUT by analog signals or via communication protocols, through the Communication Bus. As the PHIL Interface, CHIL Interface also must have hard-real-time requirements. In addition, some applications may require amplifiers to provide higher analog signals of voltages and/or currents than those provided by the RTS platform interface. In such applications, there is the necessity to apply two-quadrant (2Q) amplifiers that acts only as a voltage or current sources. Since amplifiers are not charged to provide or sink power to/from DUT, unlike PHIL, the performance of the amplifier is not so critical in the CHIL applications.

\section{E. Communication Bus}

The communication Bus has two main functions: to communicate all equipment in the proposed platform and to provide an emulated communication network to represent different topologies and scenarios of studies. With respect to the first function, the communication between RTS platform and another control device are realized by the Communication Bus. For this reason, this bus must have hard-real-time requirements, in order to guarantees the minimal latency in the communication process. On the other hand, the second function is related to improve the flexibility of the proposed laboratorial platform, where will be possible to emulate several topologies, types of physical environments and communication protocols usually adopted in microgrids applications. By this approach, the user will be able to perform analyses in the communication system under different scenarios involving modification throughput, delay, latency, and accuracy of the processed information.

\section{F. RTS Platform (Simulated Environment)}

The RTS platform provides the interface between the simulated digital model and physical electrical devices, 
both in the control and the power level. With respect to the simulated environment, all the components that are not physical devices must be properly represented in the digital model. As earlier discussed, the simulated model must be computed in hard-real-time requirements, mainly in terms of deterministic and small time-step of the simulation.

With respect to the actual infrastructure of the LASSE, the laboratory has four RTDS ${ }^{\circ}$ GPC-based racks. However, in this year this old structure will be changed to the NovaCor RTDS $®$ platform. The choice of RTDS® products is motivated by four main reasons: i) the experience of the team in this platform, due to the fact that the LASSE works by ten years with this simulator; ii) the RTDS® keep an actualized library, with constant improvements of distribution and renewable energy models; iii) RTDS® has a dedicated communication link to interface with the 4Q amplifiers of some manufacturers, in order to improve the stability and accuracy of the PHIL simulations; and iv) there is the possibility to implement specific models that are not belonging to the default library through the CBuilder environment, adding flexibility to the performed analyzes.

\section{The Capability of Analysis of the Proposed Laboratorial Infrastructure}

With the proposed infrastructure will be possible to perform a lot of analysis to fulfill the gaps from technical and regulatory viewpoints regarding the microgrid concept. In general guidelines, the evaluation of a microgrid in a laboratorial environment allows tests under various operating conditions, including the worst-cases, without impacting the grid devices and even the connected customers [14]. Among the subjects that can be addressed by the proposed infrastructure, the followings stand out:

1) Control and Management of DERs: testing of algorithms and control laws for managing local resources (e.g. renewable sources, generation and stored energy) and loads of the evaluated microgrid, considering both grid-connected and islanded modes of operation [14] . In this subject, it will be possible to analyze the dispatch of units under supply and demand uncertainty, to determine the appropriate levels of reserves to maintain the reliability indexes of microgrids inside adequate values. Furthermore, it will be possible to seek guidelines to obtain a reliable and an economical operation of these systems even in the cases with high penetration levels of intermittent generation;

2) Impacts of DERs over DPSs: measure the impacts of the connection of different generation topologies -with dispatchable and nondispatchable characteristics - and energy storage systems over the performance of DPSs considering different topologies;

3) DC Microgrids Analyses: due to the presence of the 4Q amplifiers and RTS platform as well as a DC bus, it will be possible to perform the testing of microgrids in which sources and loads are of DC type. This class of microgrid is of common application in maritime and aeronautical applications. For this reason, this kind of analysis presents great interest, especially by military agencies and manufacturers of aircraft and vessels;

4) Power Electronics: testing of different topologies and controls applied to the power electronic interfaces of renewables as well as storage systems, checking aspects from the performance and efficiency viewpoints;

5) Communications: evaluate different proprietary communication protocols in the microgrid performance, and the effects of the malfunction of communication systems over the microgrid operation. In this same subject, it will be possible the proposal of newer communication protocols for microgrid applications;

6) Regulatory Support: as in a worldwide level, the microgrid is a relatively new subject for the BES. In this context, the proposed infrastructure can be used for the formulation of standards for the operation and establishment of microgrids;

7) Demand Side Management (DMS) Proposals: design of appropriate DMS schemes to allow customers - with the endorsement of utility - to react to the microgrid conditions of generation and stored energy, as well as the proposal of new architectures and testing of smart meters. The active participation of the local customers is beneficial to microgrid environment, especially for those that operate isolated from the bulk power systems [14];

8) Cybersecurity: evaluate the impacts of cybersecurity aspects over the microgrid performance and reliability. Furthermore, it will be possible the proposal and testing of firewalls architectures to avoid problems coming from the cybersecurity nature;

9) Islanding and Resynchronization Concerns: testing and proposal of algorithms for the identification of islanded operation condition, as well as for the resynchronization process, considering different compositions of generation technologies, grid topologies and loads;

10) Protection: testing of functions and parametrization conformance of classical protection systems applied to microgrids. In this same subject, it will be possible to propose new algorithms adhering to the reality of microgrids, especially of those with a high penetration of electronically interfaced resources;

11) Steady-state and Dynamic Performance Assessment: to perform analysis from the systemic viewpoint, obtaining the characterization of the interaction between the elements of microgrids. By this assessment, it will be possible to obtain the voltage profile of the system, short-circuit levels, losses, and the dynamic interaction between the devices from the small-disturbance and non-linear viewpoints, especially in systems with high penetration of 
electronically interfaced resources, which might show a low-inertia characteristic [6] ;

12) Power Quality: analysis of power quality aspects, specifically in systems with a high penetration of DERs electronically interfaced with the grid and with the presence of loads with non-linear characteristics;

13) Tests of Conformance: performing tests to verify the conformance of devices (e.g. inverters) to the actual standards of interconnection and good engineering practices;

14) Training Platform: training achievements in microgrids subjects, especially from the operation viewpoint, for employees of the electricity sector companies, scholars and researchers.

\section{Conclusion}

The microgrid concept constitutes an important characteristic of the future electrical power systems. The movement towards this trend is due to the necessity of the use of the energy resources in a more intelligent and efficient way, as well as by the constant growth of automation and integration of communication and control systems in the DPSs. By these arguments, it is mandatory the knowledge and the domain of the related subjects for the creation of national critical mass in this concept. In this context, this work makes your contribution via the proposition of a laboratorial infrastructure dedicated to the analysis of microgrids systems. With the forecast to implement at the end of this year in the actual facilities of LASSE in the Itaipu Technological Park Foundation Brazil, the proposed infrastructure congregates the principal characteristics of the facilities verified in international institutes and laboratories of reference in this subject. As its main differential can be highlighted the flexibility of analysis added by the presence of RTS platform with the use of the concept PHIL, as well as emulated and real elements, which can be operated together or even, depending upon on the necessities, in an isolated way. It should be highlighted the fact that at this moment it is provisioned only the analysis of pure electrical microgrids. Depending on your developments, in the future trends of the work, with the aim of following global trends, it is provisioned the integration of heat and water elements to perform more complete and complex analysis.

\section{Acknowledgement}

We are grateful for the support of Itaipu Binacional and this work is part of the research group "Microgrids and Systems with Distributed Generation by Real Time Simulation" (CNPQ) and "Hybrid Intelligent Electrical Microregions with High Penetration of Renewable Energies" (CYTED-717RT0533).

\section{References}

[1] B. Lundstrom, M. Shirazi, M. Coddington and B. Kroposki, "An Advanced Platform for Development and Evaluation of Grid Interconnection Systems Using Hardware-in-the-Loop: Part III -- Grid Interconnection System Evaluator," 2013
IEEE Green Technologies Conference (GreenTech), Denver, CO, 2013, pp. 392-399.

[2] A. Keyhani, A. Design of Smart Power Grid Renewable Energy Systems, Volume 1, New York: Wiley-IEEE Press, 2011, p. 592.

[3] J. Driesen and R. Belmans, "Distributed Generation: Challenges and Possible Solutions," 2006 IEEE Power Engineering Society General Meeting, Montreal, Que., 2006, p. 1-8.

[4] R. H. Salim, Uma Nova Abordagem para a Análise da Estabilidade a Pequenas Perturbações em Sistemas de Distribuição de Energia Elétrica com Geradores Síncronos Distribuídos (in portuguese). Thesis in Electrical Engineering, University of São Paulo, São Carlos, 2011.

[5] DOE, Summary Report: 2012 Microgrid Workshop Report, Office of Electricity Delivery and Energy Reliability Smart Grid R\&D Program, Chicago, Illinois, 2012.

[6] D. E. Olivares et al., "Trends in Microgrid Control," in IEEE Transactions on Smart Grid, vol. 5, no. 4, July 2014, pp. 1905-1919.

[7] CIGRE. Working Group C6.22 Microgrids Evolution Roadmap, Microgrids 1 Engineering, Economics, \& Experience. 2015.

[8] A. Bani-Ahmed and A. Nasiri, "Development of RealTime Hardware-in-the-Loop Platform for Complex Microgrids," 2015 International Conference on Renewable Energy Research and Applications (ICRERA), Palermo, 2015, pp. 994-998.

[9] N. Hatziargyriou, H. Asano, R. Iravani and C. Marnay, "Microgrids," in IEEE Power and Energy Magazine, vol. 5, no. 4, pp. 78-94, July-Aug. 2007.

[10] M. S. Ortmann et al., "Architecture, Components and Operation of an Experimental Hybrid AC/DC Smart Microgrid," 2017 IEEE 8th International Symposium on Power Electronics for Distributed Generation Systems (PEDG), Florianopolis, 2017, pp. 1-8.

[11] NREL, "ESIF 2017" Modernizing Our Grid and Energy System. Energy Systems Integration Facility (ESIF) 2017 Annual Report, Golden, CO: National Renewable Energy Laboratory.

[12] P. Dondi, et al. "Network Integration of Distributed Power Generation". Journal of Power Sources, vol. 106, issues 12, 2002, pp 1-9.

[13] J.A. P. Lopes, "Integrating Distributed Generation into Electric Power Systems: A Review of Drivers, Challenges and Opportunities", Electric Power Systems Research, vol. 77, Issue 9, 2007, pp. 1189-1203.

[14] A. Maitra et al., "Microgrid Controllers: Expanding Their Role and Evaluating Their Performance," in IEEE Power and Energy Magazine, vol. 15, no. 4, pp. 41-49, July-Aug. 2017.

[15] RTDS. Power Hardware in the Loop Simulation (PHIL), Real Time Digital Simulator PHIL Report, Canada, 2018, p. 42.

[16] W. Ren, M. Steurer and T. L. Baldwin, "Improve the Stability and the Accuracy of Power Hardware-in-theLoop Simulation by Selecting Appropriate Interface Algorithms," in IEEE Transactions on Industry Applications, vol. 44, no. 4, pp. 1286-1294, July-Aug. 2008.

[17] J. Wang, Y. Song, W. Li, J. Guo and A. Monti, "Development of a Universal Platform for Hardware Inthe-Loop Testing of Microgrids," in IEEE Transactions on Industrial Informatics, vol. 10, no. 4, pp. 2154-2165, Nov. 2014. 